\title{
A alimentação de juvenis de matrinxã, Brycon amazonicum (Pisces, Characidae), em áreas inundadas da Ilha de Marchantaria, Amazonas, Brasil.
}

\author{
Rosseval Galdino LEITE ${ }^{1}$
}

\begin{abstract}
RESUMO
Estudou-se a dieta de 44 exemplares de juvenis de Brycon amazonicum (de 15 a 50mm de C.P), em áreas inundadas da Ilha de Marchantaria, no rio Solimões. Os peixes foram capturados com rede-de-mão com $30 \mathrm{~cm}$ de diâmetro e cabo de 2 metros de comprimento. As análises foram baseadas em Freqüência de Ocorrência (\%) e no número de presas. A dieta foi analisada pelo tamanho (C.P. (mm)) e por data de captura usando uma ANOVA com dois fatores. A data de captura explicou a oscilação no número de presas ingeridas pelos juvenis. A análise qualitativa mostrou poucas mudanças na dieta nesta fase de desenvolvimento. Verificou-se que B. amazonicum apresenta amplo espectro alimentar e que regula o número de presas na dieta de acordo com a disponibilidade dessas presas no ambiente.
\end{abstract}

PALAVRAS-CHAVES

Amazônia, peixes, Brycon amazonicum, alimentação, áreas inundadas.

\section{The diet of matrinxã Brycon amazonicum (Pisces, Characidae) juveniles, in flooded areas of the Marchantaria Island, Amazonas, Brazil.}

\begin{abstract}
The diet of 44 Brycon amazonicum juveniles (15 to $50 \mathrm{~mm}$ S.L.) from the flooded areas of the Marchantaria Island in the Solimões River was studied. The fishes were captured using a $30 \mathrm{~cm}$ diameter aperture and 2 meter long band net. Our analyses were based on Frequency Occurence (\%) and prey number. Larvae diet was evaluated by length (S.L (mm)) and date of capture, using two factors ANOVA. The dates of capture explain the oscillations in the prey number ingested by juveniles. The qualitative diet shows little variation among juveniles of Brycon amazonicum during this development phase. In conclusion, B. amazonicum juveniles present a large food spectrum and adjust their diet according to prey availability in the environment.
\end{abstract}

KEY WORDS

Fish, feeding, Brycon amazonicum, Amazon, floodplain
O gênero Brycon é representado por espécies comercialmente importantes, e muito apreciadas pela população da Amazônia Central (Petrere, 1978; Smith, 1979, 1981; Goulding, 1979, 1980, 1981). As informações sobre a dieta de peixes do gênero Brycon, principalmente adultos, e de jovens maiores que $60 \mathrm{~mm}$, mostram a importância dos alimentos de origem vegetal e artrópodes (Muller \& Troschel, 1844; Breeder, 1927; Menezes, 1969; Knöpell, 1970; Goulding, 1980; Borges, 1986).

Em trabalhos recentes, a espécie que aqui denominamos de Brycon amazonicum foi erroneamente considerada como Brycon cephalus. Entretanto, a distribuição de $B$. cephalus restringe-se ao alto rio amazonas no Peru e na Bolívia (Lima, 2003). As informações sobre juvenis iniciais de B. amazonicum em condições naturais são escassas, e isto ocorre porque os apetrechos utilizados na maioria dos estudos não são adequados para a sua captura, principalmente porque nas primeiras fases de desenvolvimento, os indivíduos desta espécie se encontram entre as macrófitas aquáticas.

A alimentação natural das larvas de B. amazonicum é mais bem conhecida pelo trabalho de Leite \& Araújo-Lima

${ }^{1}$ Instituto Nacional de Pesquisas da Amazônia 


\section{ACTA AMAZONICA}

A ALIMENTAÇÃO DE JUVENIS DE MATRINXÃ, Brycon amazonicum (PISCES, CHARACIDAE), EM ÁREAS INUNDADAS DA ILHA DE MARCHANTARIA, AMAZONAS, BRASIL
(2002). Nesta fase de desenvolvimento, a espécie alimentase de zooplâncton e, principalmente, de larvas de outras espécies de peixes.

O presente trabalho descreve o que ocorreu com a dieta desta espécie em uma área inundada do rio Solimões, em indivíduos que variavam de 15 a $50 \mathrm{~mm}$ de Comprimento Padrão. A importância dessas informações para os criadores do matrinxã, como é conhecido em Manaus, reside no fato de que, nesta faixa de tamanho, os juvenis reproduzidos em estações de aqüicultura, são introduzidos nos seus sistemas de criação intensiva ou semi-intensiva.

Para este estudo foram utilizados 44 exemplares de $B$. amazonicum capturados em áreas inundadas da Ilha de Marchantaria no rio Solimões (Fig. 1), em 7 excursões realizadas em 1994: 18, 25 e 27/Jan.; 04 e 21/Fev., 13 e 19/Dez. Os peixes foram capturados aleatoriamente com uma rede-de-mão (rapixé), com aro de $30 \mathrm{~cm}$ de diâmetro e cabo de $2 \mathrm{~m}$ de comprimento. Logo após a captura, os peixes foram fixados em solução de formalina a 10\% e acondicionados em frascos de vidro, identificados por data e local. No laboratório os peixes foram repassados para uma solução de álcool a 70\% após lavagem em água corrente, para a retirada do excesso de formol.

De cada peixe foram tomadas medidas de comprimento padrão, e somente o conteúdo estomacal foi analisado após a retirada do estômago da cavidade abdominal dos peixes. Os alimentos de origem vegetal foram divididos em sementes e/ou frutos e restos de vegetais (folhas, raízes ou caules). Os alimentos de origem animal foram identificados

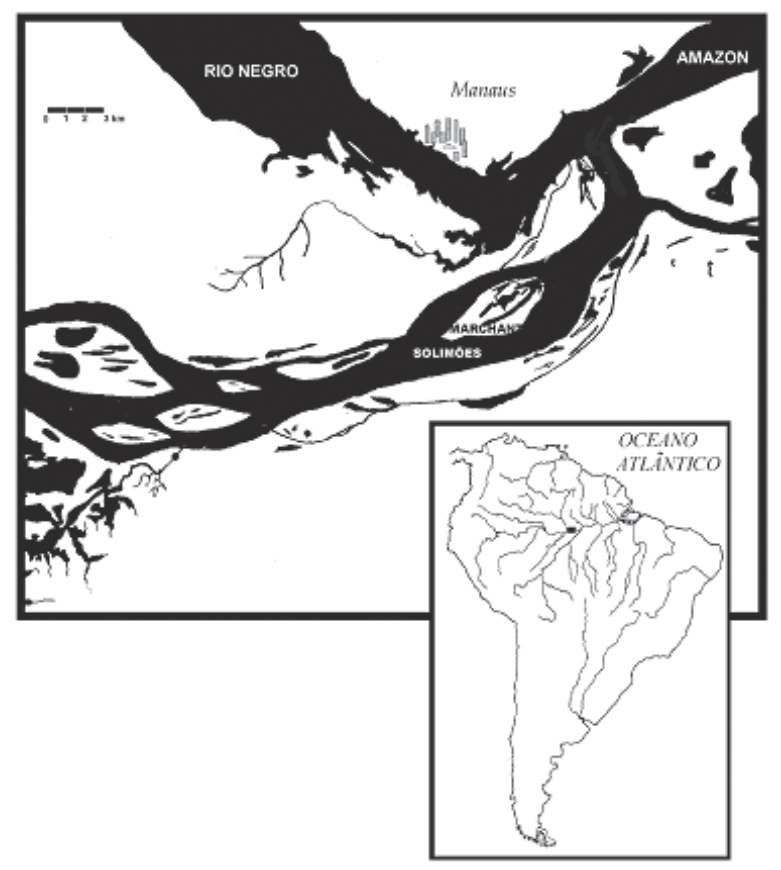

Figura 1 - Área de captura dos juvenis de Brycon amazonicum, com destaque para a Ilha de Marchantaria e sua localização no rio Solimões e posição em relação a América do Sul e cidade de Manaus-AM. em nível de grandes grupos como pertencentes a: Rotifera, Copepoda, Cladocera, Insecta (imaturos e adultos) e peixes (larvas, músculos e escamas).

Para descrever qualitativamente a dieta dos juvenis, utilizouse o Método de Freqüência de Ocorrência (\%) (Hyslop, 1980), que considera o número de vezes que um determinado item ocorre em relação ao total de indivíduos com alimento no estômago. Para avaliar variações no número de presas comidas, foram feitas Análises de Variância com dois fatores, da quantidade $(\mathrm{Y})$ das presas, em relação ao tamanho dos juvenis (C.P. (mm)), e também com as datas de captura.

Os juvenis de Brycon amazonicum pouco alteraram a sua dieta em função do tamanho, tanto qualitativa quanto quantitativamente. Mais de $96 \%$ dos juvenis com tamanhos de 15 até $25 \mathrm{~mm}$ comeram Micro-crustáceos (Cladocera, Copepoda) e Rotifera, baixando para cerca de $80 \%$ a partir de $25 \mathrm{~mm}$ de C.P. O item Restos vegetais foi encontrado em praticamente todos os indivíduos examinados e houve um ligeiro acréscimo no número de indivíduos comendo Sementes, nos maiores que $25 \mathrm{~mm}$ de C.P.

Somente o item Escama e "Outros" alimentos (músculo de peixes, nematóides), foram comidos por cerca de $20 \%$ dos indivíduos de tamanhos variando entre 25 e $50 \mathrm{~mm}$ e que não foram detectados nos estômagos dos indivíduos menores (Figuras 2A e 2B).

A maior fonte de variação no número de presas individuais ingeridas pelos juvenis de $B$. amazonicum nas
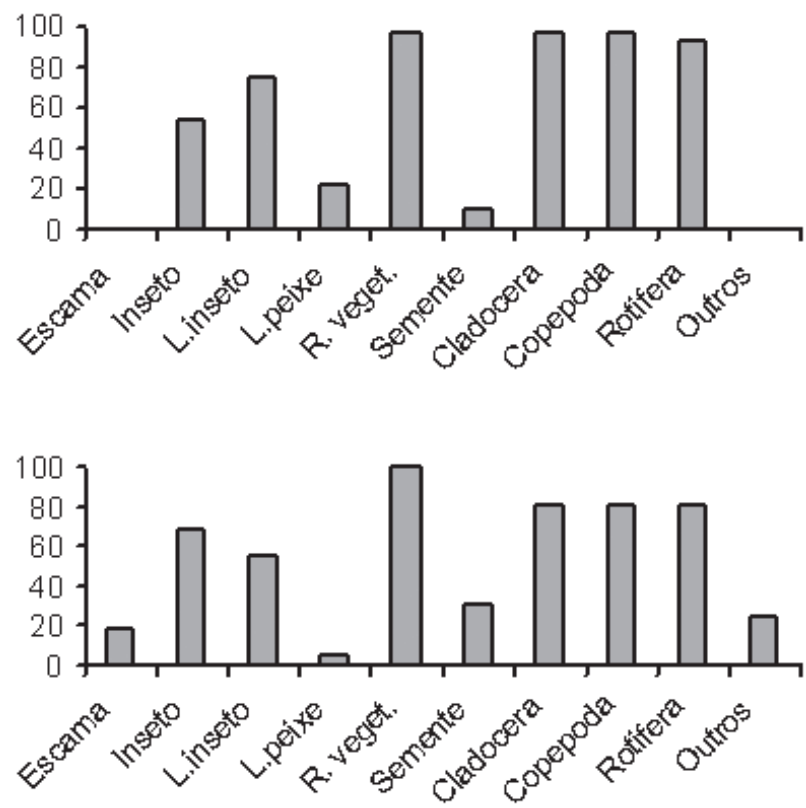

Figura 2 - Gráficos da Freqüência de Ocorrência (\%) dos itens alimentares encontrados no estômago de Brycon amazonicum, de 15 a $25 \mathrm{~mm}(\mathbf{A})$ e 25 a $50 \mathrm{~mm}$ (B) de Comprimento Padrão capturados em áreas inundadas da Ilha de Marchantaria nos meses de janeiro, fevereiro e dezembro de 1994. 


\section{ACTA AMAZONICA}

interações entre tamanho do peixe e data de captura foi o fator data de captura (Tabela 1).

O pequeno número de juvenis de Brycon amazonicum avaliados neste trabalho, deveu-se ao fato da dificuldade da sua captura com rede-de-mão, tendo em vista que nesta fase, principalmente os exemplares maiores dentre os capturados, encontram-se entre as macrófitas aquáticas e são muito velozes. Mesmo assim, consideramos que os dados aqui apresentados são consistentes e refletem o comportamento natural da espécie nesta fase de desenvolvimento.

$\mathrm{O}$ hábito de comer larvas de peixes nas primeiras fases

Tabela 1 - Estatísticas das Análises de Variância (modelo misto) da quantidade $(\mathrm{Y})$ das presas por tamanho e data de captura, dos juvenis de Brycon amazonicum em áreas inundadas na Ilha de Marchantaria no rio Solimões/Amazonas, nos meses de Dezembro, Janeiro e Fevereiro de 1994.

\begin{tabular}{|c|c|c|c|c|}
\hline Itens/fontes de variação & G.L. & QM & $\mathbf{F}$ & $\mathbf{P}$ \\
\hline \multicolumn{5}{|l|}{ Escamas } \\
\hline Data & 5 & 0,485 & 0,156 & 0,986 \\
\hline C.P. $(\mathrm{mm})$ & 1 & 6,411 & 0,354 & 0,990 \\
\hline Interação & 6 & 0,764 & 0,246 & 0,939 \\
\hline Erro & 31 & 3,106 & & \\
\hline \multicolumn{5}{|l|}{ Insetos } \\
\hline Data & 5 & 3,560 & 1,162 & 0,350 \\
\hline C.P. $(\mathrm{mm})$ & 1 & 4,812 & 1,571 & 0,219 \\
\hline Interação & 6 & 3,880 & 1,267 & 0,301 \\
\hline Erro & 31 & 3,063 & & \\
\hline \multicolumn{5}{|l|}{ Larvas de insetos } \\
\hline Data & 5 & 7,180 & 0,553 & 0,764 \\
\hline C.P. $(\mathrm{mm})$ & 1 & 6,411 & 0,354 & 0,990 \\
\hline Interação & 6 & 4,524 & 0,349 & 0,905 \\
\hline Erro & 31 & 12,979 & & \\
\hline \multicolumn{5}{|l|}{ Larvas de peixes } \\
\hline Data & 5 & 5,531 & 3,271 & 0,017 \\
\hline C.P. $(\mathrm{mm})$ & 1 & 1,443 & 0,449 & 0,963 \\
\hline Interação & 6 & 4,837 & 2,861 & 0,025 \\
\hline Erro & 31 & 1,691 & & \\
\hline \multicolumn{5}{|l|}{ Cladocera } \\
\hline Data & 5 & 138,665 & 2,408 & 0,059 \\
\hline C.P. $(\mathrm{mm})$ & 1 & 67,679 & 0,542 & 0,917 \\
\hline Interação & 6 & 149,210 & 2,591 & 0,032 \\
\hline Erro & 31 & 57,590 & & \\
\hline \multicolumn{5}{|l|}{ Copepoda } \\
\hline Data & 5 & 111,536 & 0,805 & 0,154 \\
\hline C.P. $(\mathrm{mm})$ & 1 & 52,731 & 1,703 & 0,376 \\
\hline Interação & 6 & 140,584 & 2,146 & 0,086 \\
\hline Erro & 31 & 65,46 & & \\
\hline \multicolumn{5}{|l|}{ Rotifera } \\
\hline Data & 5 & 66,516 & 1,894 & 0,029 \\
\hline C.P. $(\mathrm{mm})$ & 1 & 183,374 & 5,221 & 0,124 \\
\hline Interação & 6 & 46,161 & 1,314 & 0,280 \\
\hline Erro & 31 & 35,120 & & \\
\hline
\end{tabular}

de desenvolvimento observado por Leite \& Araújo-Lima (2002), permanece ainda nos peixes juvenis de Brycon amazonicum. Este comportamento é favorecido pelo tamanho de sua boca que, na fase larval corresponde em média, a 20\% do seu comprimento padrão (Leite, 2000).

A maior intensidade da reprodução dos peixes no início da enchente do rio Solimões/Amazonas, de acordo com Araújo-Lima (1984), contribui muito para a presença de larvas de peixes na dieta das larvas de B. amazonicum que são maiores e desenvolvem-se muito mais rápido do que a maioria das larvas de Characiformes (Araújo-Lima \& Donald, 1988).

Após a fase larval, os juvenis de $B$. amazonicum ainda persistiram comendo larvas de peixes, porém em menor quantidade do que suas larvas. Neste caso, o fator tempo explicou esse comportamento, uma vez que, normalmente, há uma diminuição da atividade reprodutiva dos Characiformes e Siluriformes, na medida em que os meses passam, desde o início da subida do nível das águas do rio Amazonas (Araújo-Lima e Oliveira, 1998).

Um comportamento que não foi muito evidente nas fases larvais de B. amazonicum e que ocorreu com freqüência nos seus juvenis, foram os micro-crustáceos. Nas duas classes de comprimento, presas desta categoria compuseram a dieta de 96 a 80\% dos menores e dos maiores indivíduos examinados respectivamente. No caso de Cladoceros e Rotíferos também houve maior influência das datas de captura na interação, do que propriamente do tamanho dos juvenis, denotando também uma oscilação na oferta dessas presas no meio ambiente.

Os itens derivados da classe Insecta não oscilaram estatisticamente e também apresentaram estabilidade no nível qualitativo. É importante destacar que todos os peixes aqui analisados foram capturados em áreas de vegetação inundada, não havendo, portanto, a escassez de insetos aquáticos que é abundante e que utiliza-se das macrófitas aquáticas para o seu desenvolvimento (Junk, 1973).

Não se pode afirmar, pela natureza de nossas observações, se os pedaços de folha e raízes, e até mesmo as Sementes, foram comidos direta ou indiretamente pelos juvenis de B. amazonicum. Entretanto, mesmo que esses itens tenham sido comidos indiretamente, a sua presença no conteúdo estomacal demonstra que a vegetação desempenha um papel muito importante para os juvenis de B. amazonicum, quando é um fator determinante para a presença das presas (Junk, 1973).

Em todo o caso, a questão do aproveitamento dos alimentos de origem vegetal pelos juvenis de $B$. amazonicum merece uma discussão à parte, tendo em vista que, para o aproveitamento da energia proveniente desses alimentos, os peixes necessitam ter estruturas capazes de auxiliar na sua digestão (Schimidt-Nielsen, 1996; Logothetis et al., 2001).

Não se pode também, desprezar o fato de que as espécies de Brycon possuem a capacidade de quebrar e/ou triturar os vegetais na cavidade bucal, utilizando os dentes dispostos em série tríplice, espessos e multicuspidados (Muller \& Troschel, 1844; Géry, 1977). 


\section{ACTA AMAZONICA}

A ALIMENTAÇÃO DE JUVENIS DE MATRINXÃ, Brycon amazonicum (PISCES, CHARACIDAE), EM ÁREAS INUNDADAS DA ILHA DE MARCHANTARIA, AMAZONAS, BRASIL
Há fortes evidências de que juvenis de B. amazonicum de áreas inundadas da Ilha da Marchantaria, apresentam boa elasticidade com relação ao consumo de diferentes presas e que também atuam mais em função da disponibilidade das mesmas, podendo, já nesta fase de desenvolvimento, serem considerados "oportunistas".

\section{BIBLIOGRAFIA CITADA}

Araujo-Lima, C. A.R.M. 1984. Distribuição espacial e temporal de larvas de Caraciformes em um setor do Rio Solimões/ Amazonas próximo a Manaus, AM. Dissertação de Mestrado INPA/FUA, Manaus, 82p.

Araújo-Lima, C. A. R. M; Oliveira, E. C. 1998. Transport of larval fish in the Amazon. Journal of Fish Biology., 53 (suppl.A): 23-33.

Bayley, P. B; Petrere, M. 1989. Amazon Fisheries: Assessment methods, current status and management option. Can. Spec. Publ. Fish. Aquatic. Sci. 106: 385-398.

Borges, G. A. 1986. Ecologia de três espécies do gênero Brycon Muller \& Troschel,844 (Pisces-Characidae), no rio NegroAmazonas, com ênfase na caracterização taxonômica e alimentação. Dissertação de Mestrado INPA/FUA, Manaus.150p.

Breeder, C. M. 1927. The fishes of the rio Chucunaque drainage eastern Panamá. Bull. Amer. Mus. Nat. Hist. 57:91-176, 10 figs., 5 pls (Char: 113-130)

Géry, J. 1977. Characoids of the world. TFH, Neptune City. Publications. 672p.

Goulding, M. 1979. Ecologia da pesca do rio Madeira. CNPqINPA. Manaus, 172p.

Goulding, M. 1980. The Fishes and the forest: Explorations in Amazonian Natural History. University of California Press. Berkeley, California. 280p.

Goulding, M. 1981. Man and Fisheries on an Amazon Frontier. J. W. Junk Publishers, The Hague. 121p.

Hyslop, E. J. 1980. Stomach contents analysis - a review of methods and their application. Journal of Fish Biology., 17:411-429.

Junk, W. J. 1973. Investigations on the ecology and productionbiology of the "floating-meadows" (Paspalo-Echinochloetum on the Midle Amazon). II - The aquatic fauna in the root-zone of floating vegetation. Amazoniana, IV: 9-102.
Knöppel, H. A. 1970. Food of central Amazonian fishes. Contribution to the nutrient ecology of Amazonian rain-forest streams. Amazoniana 2 (3):257-352.

Leite, R. G. 2000. Alimentação e fontes autotróficas de energia das larvas de peixes no rio Solimões/Amazonas e suas áreas inundáveis. Tese de Doutorado INPA/FUA, 118p.

Leite, R. G.; Araujo-Lima, C.A.R.M. 2002. Feeding of the Brycon amazonicum, Triportheus elongatus and Semaprochilodus insignis (Osteichthyes, Caraciformes), larvae in Solimões/ Amazonas river and floodplain areas. Acta Amazonica 3(2):56-67.

Lima, F. C. T. 2003. Subfamily Bryconinae (Characins, Tetras). In: Reis, R.E; Kulander, S. O; Ferraris Jr, C. J. (Orgs.) Check List of the Freshwater Fishes of South and Central America. EDPURCS, Porto Alegre: 174-181.

Logothetis, E.A; Horn, M. H; Dickson, K.A. 2001. Gut morphology and function in Atherinops affinis (Teleostei:Atherinopsidae), a stomachless omivore feeding on macroalgae. J. Fish Biol. 59, 1298-1312.

Menezes, N. A. 1969. The food of Brycon and three closely related genera of the tribe Acestrorhynchini. Papeis Avulsos de Zoologia. SP. 22 (20):217-223.

Müller, J.; Troschel, F. H. 1844. Synopsis generum et specierum familiae characinorum (Prodromus descriptions novorum generum et specierum). Archiv. Naturgesch., 10(1):81-99.

Petrere, M. 1978. Pesca e esforço de pesca no estado do Amazonas. II. Locais, aparelhos de captura e estatísticas de desembarque. Acta Amazonica 8, suppl. 2: 54pp.

Smidt-Nielsen, K. 1996. Fisiologia Animal: Adaptação e meio ambiente. Livraria Santos Editora, São Paulo, Brasil. 600p.

RECEBIDO EM: 02/09/2003

ACEITO EM: 17/09/2004 